Vietnam Journal of Mechanics, VAST, Vol. 42, No. 3 (2020), pp. 321 -336

DOI: https://doi.org/10.15625/0866-7136/15479

Dedicated to Professor J.N. Reddy on the Occasion of His $75^{\text {th }}$ Birthday

\title{
PLASTICITY BASED INTERFACE MODEL FOR FAILURE MODELLING OF UNREINFORCED MASONRY UNDER CYCLIC LOADING
}

\author{
P. V. S. K. Kumar ${ }^{1}$, Amirtham Rajagopal ${ }^{1, *}$, Manoj Pandey ${ }^{2}$ \\ ${ }^{1}$ Department of Civil Engineering, Indian Institute of Technology Hyderabad, India \\ ${ }^{2}$ Department of Mechanical Engineering, Indian Institute of Technology Madras, India \\ *E-mail: rajagopal@iith.ac.in
}

Received: 09 July 2020 / Published online: 27 September 2020

\begin{abstract}
In this work our objective is to understand the failure behaviour of unreinforced masonry under in-plane cyclic loading. For this purpose we proposed a plasticity based interface model consists of a single yield surface criteria which is a direct extension of Mohr-Coulomb criteria with a tension cut and compression cap and a back stress vector is introduced as a mixed hardening law variable in the adopted yield surface to capture the unloading/reloading behaviour of masonry under cyclic loading. A simplified micromechanical interface modelling approach is adopted to capture all the failure modes of masonry. The integration of the differential constitutive equation is done by using implicit Euler backward integration approach and the obtained non-linear set of equations are solved by a combined local/global Newton solver. The proposed constitutive model is implemented in ABAQUS by writing UMAT (user-defined subroutine) and the obtained numerical results are compared with experimental results available in the literature.
\end{abstract}

Keywords: plasticity interface model, single yield surface, back stress vector, simplified micro model.

\section{INTRODUCTION}

Unreinforced masonry (URM) structures are very common constructions found in rural parts of India. Such structures are sensitive and susceptible to damage in the event of any magnitude of seismic action. Understanding the behaviour of such URM structures has therefore become important [1]. Such studies will allow to arrive at efficient designs of such URM constructions. There are different patterns of arrangement of bricks and mortar in an URM construction, that makes them heterogeneous and anisotropic. In last few decades, there has been several works to model and understand the complex nonlinear behavior of URM structures under cyclic loads. The study amounts to understand the interface behavior between the brick and mortar [2]. From this perspective several nonlinear interface models have been developed to model masonry in a finite element numerical setting [3]. Macro- [4], Meso- [5], Micro- [6], Macro-micro [7] approaches have commonly been used to model the URM structures.

Simplified micro modeling approaches are more appealing and popular because of their ability to capture true behavior, including the effects of brick and mortar locally and at their contact $[8,9]$. In simplified micro modelling bed and head mortar joint interface are modelled as zero-thickness discontinuous inelastic interface elements and expanded brick units are modelled with elastic continuum elements [10]. Such simplified micro models are able to represent all possible modes of failure namely: masonry unit direct tensile cracking, masonry joint tensile cracking, masonry joint slipping, masonry unit diagonal tensile cracking and crushing in masonry [9].

There has been several experimental and numerical works in understanding the behaviour of URM walls under cyclic loading [11,12]. It is observed from experimental studies that there is a stiffness a degradation in both tension as well as compression, however no degradation is observed in direct 
shear stiffness [13]. From the experimental study it is noted that since there is a stiffness degradation in normal loading and no stiffness degradation under shear loading, non-linear and linear elastic material behaviour must be considered under normal and shear loading respectively. There have been several models proposed in literature to predict the stiffness degradation in URM using concepts from fracture, damage mechanics and plasticity theories (see [2,14-18]). Modified Mohr-Columb failure criteria with isotropic and kinematic hardening in the frame work of non associative plasticity have been proposed to understand the failure behaviour under monotonic loading. Distinct criterias for shear compression $[19,20]$, and shear tension regions [21] are incorporated in such models.

There has been several works on modelling behavior of URM walls under cyclic loads. Lorenco et al. [10] used interface multi-surface yield criteria where the three yield functions are used to simulate the failure of masonry for various loading like tension, compression and shear. The drawback in this model is the singularity issue at the transition zone from tension to shear and shear to compression. Oliveira et al. [22] extended the Lorenco et al. [10] model by introducing two unloading yield surfaces (unloading to tension and compression) to understand failure behaviour of masonry subjected to cyclic loading. In this model, it is assumed that non-linearity occurs at the interface elements while all the elements in this model are assumed to remain in elastic region. The model is able to capture the non-linear failure behaviour of masonry subjected to in-plane monotonic and cyclic loads. To simulate sliding and separation of brick under cyclic load a cohesive-friction contact based formulation is implemented obeying damage plasticity constitutive law in tension and compression. The contact behaviour is governed by Mohr-Coulomb criteria with tension cutoff [23]. A hybrid numerical method having a combination of finite elements for brick units and discrete element for interface is proposed in [24]. The failure behaviour of masonry wall under reversed cyclic test has been predicted. The model is able to capture opening and sliding behaviour of masonry elements. The main drawback in this model is that crushing of masonry as a possible failure mode under cyclic loads is not considered in the analysis. In [5], the evolution of anisotropic damage tensor coupled with plastic work is introduced to understand the behaviour of masonry wall under cyclic loading. The plastic work corresponding to each fracture mode is calculated to produce the post-peak (softening) behaviour of masonry. Non-local damage plasticity approach is capable to capture stiffness degradation/recovery during loading/unloading has been proposed in [25]. The model is found to be very useful to predict the behavior of URM walls under quasi-static and cyclic loads. The effect of multi-layer bed joints on shear behavior of unreinforced masonry under cyclic loading has been studied in [26]. The elastic and inelastic constitutive material models have been proposed in [27] to capture linear and non-linear behaviour of masonry subjected to cyclic loadings. A user defined subroutine for ABAQUS has been developed. From the overview of literature it is observed that most models are based on multisurface plasticity theories and incorporating isotropic hardening together with some stiffness degradation function or recovery functions for loading/unloading or reloading cycles.

In this work we present a simplified micromodeling approach for modeling the URM walls subjected to cyclic loads. A plasticity based composite interface model is proposed and formulated. The proposed plasticity models incorporates (i) a single surface yield criteria devoid of any singularities at the corners and representing all modes of failure, (ii) a mixed hardening law that accounts for stiffness and strength degradation/recovery during unloading/reloading and (iii) a set of internal hardening variables whose evolution is based on energy based hardening parameters corresponding to particular modes of failure. The mixed hardening is introduced via a mixed hardening parameter that accounts for the proportions of isotropic and kinematic hardening at any stage of loading. A back stress vector is defined to incorporate the change in origin of the yield surface because of kinematic hardening and it introduces yield surface with combined hardening. The proposed single surface criterion was earlier tested for performance under monotonic loads and isotropic hardening [9] and has been extended to cyclic loads with mixed hardening in the present work.

This paper is organized as follows. In Section 2, the proposed plasticity based composite interface model for URM wall subjected to cyclic loading is presented. Various components of model like 
single surface yield function, hardening parameters, mixed hardening rule, derivation of elasto-plastic tangent moduli and elastic plastic constitutive relations are discussed. In Section 3 the numerical implementation and algorithm implementation for the proposed model is proposed. In Section 4 numerical examples are presented to demonstrate the effectiveness of the proposed model. In the last section we present the summary and conclusions.

\section{PROPOSED PLASTICITY BASED COMPOSITE INTERFACE MODEL}

In this section we present the proposed plasticity based composite interface model for URM wall subjected to cyclic loading. In simplified micro modeling bed and head mortar joint interface are modelled as zero-thickness discontinuous inelastic interface elements and expanded brick units are modelled with elastic continuum elements [10]. Various components of model are discussed below:

\section{Elastic stress-strain relationship}

The elastic stress and strain at the interface are related as $\sigma=\mathbf{K} \epsilon$, where $\sigma=\left[\sigma_{n n}, \sigma_{t t}\right]^{T}$, and per unit displacements are given by $\epsilon=\left[u_{n n}, u_{t t}\right]^{T}$. $n n$ and $t$ represent the normal and tangential terms. The effect of Poisson's ratio for the interface is assumed to be negligible. The stiffness matrix is given by $K=\operatorname{diag}\left[k_{n n}, k_{t t}\right]$, where $k_{n n}$ and $k_{t t}$ represents normal and tangent stiffness. The units are assumed to be linear elastic due to large difference in the thickness of unit and mortar. The elastic stiffness matrix components $\left(k_{n n}, k_{t t}\right)$ are represented as $\frac{1}{K_{n n}}=\frac{1}{h_{m}}\left(\frac{1}{E_{u}}+\frac{1}{E_{m}}\right)$ and $\frac{1}{K_{t t}}=\frac{1}{h_{m}}\left(\frac{1}{G_{u}}+\frac{1}{G_{m}}\right)$. Where $E_{u}, E_{m}$ and $G_{u}, G_{m}$ represent the Young's modulus and the Shear modulus of the brick units and mortar respectively.

\section{Rate independent plasticity}

In the present study a rate independent composite interface model, defined by hyperbolic function (Eq. (1)) has been proposed (see Fig. 1). The proposed model is a simple extension of the Mohr-Coulomb criteria with cut-off in tension and cap-off in compression, which result in the single surface yield criteria capable of representing pressure-dependent friction shear failure and cracking by cut-off in-tension and crushing by cap-off in compression under combined normal and tangential stresses. The model includes all failure mechanisms of masonry and it also overcomes the problem of the singularity that occurs in multi-surfaces yield criteria. The proposed model is for a case of combined hardening and includes both the isotropic and

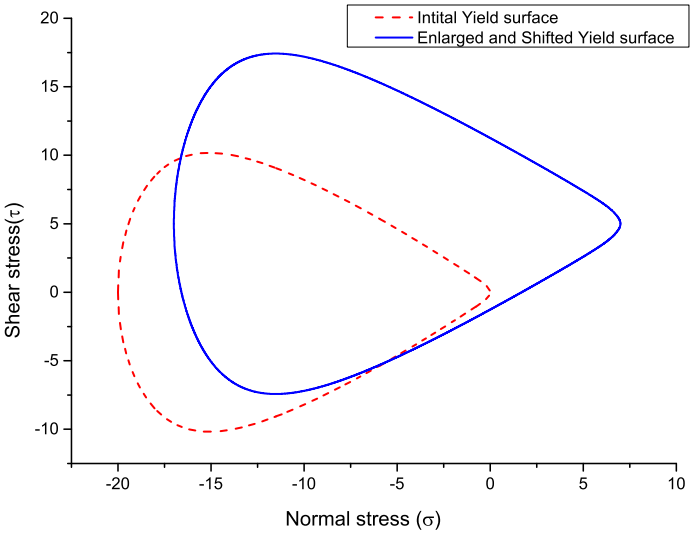

Fig. 1. Mixed hardening kinematic hardening parts. The kinematic hardening part is specifically introduced in the present formulation through the definition of a kinematic hardening variable, that allows to define inelastic unloading/reloading behavior and helps in defining the back stress vector (a). The single surface hyperbolic function for the mixed hardening case is given by

$$
F(\sigma, \mathbf{q}, \mathbf{a}):=-\left[C-\tilde{\sigma}_{n n} \tan (\phi)\right]^{2} f_{c}(\sigma, \mathbf{q}, \mathbf{a}) f_{t}(\sigma, \mathbf{q}, \mathbf{a})+\tilde{\sigma}_{t t}^{2},
$$

where $f_{c}(\sigma, \mathbf{q}, \mathbf{a}):=\frac{2}{\pi} \arctan \left(\frac{\tilde{\sigma}_{n n}-\zeta}{\alpha_{c}}\right)$ and $f_{t}(\sigma, \mathbf{q}, \mathbf{a})=\frac{2}{\pi} \arctan \left(\frac{\xi-\tilde{\sigma}_{n n}}{\alpha_{t}}\right)$. The reduced stress is defined as $\tilde{\sigma}=\sigma-\boldsymbol{a} . f_{t}(\sigma, \mathbf{q}, \mathbf{a})$ and $f_{c}(\sigma, \mathbf{q}, \mathbf{a})$ defines the tension cut-off and compression cap-off functions respectively. Function $f_{t}(\sigma, \mathbf{q}, \mathbf{a})$ and $f_{c}(\sigma, \mathbf{q}, \mathbf{a})$ become zero at tension cut $(\xi)$ and compression cap $(\zeta)$ respectively. For all other stress states, the functions $f_{t}(\sigma, \mathbf{q}, \mathbf{a})$ and $f_{c}(\sigma, \mathbf{q}, \mathbf{a})$ are approximately equal to one. The curvature of the compression cap and tension cut-off at transition region are controlled by the parameter $\alpha_{c}$ and $\alpha_{t}$ respectively. The hardening parameter $\left(\mathbf{q}=\mathbf{q}\left(C, C_{q}, \phi, \psi, \xi, \zeta\right)\right)$ is a function of cohesion $C$, apparent cohesion $C_{Q}$, dilation angle $\phi$, friction angle $\psi$, tensile strength $\xi$ 
and compressive strength $\zeta$. Following an non associative flow rule, the plastic potential is defined in a non-associative formulation which consists of apparent cohesion $\left(C_{q}\right)$ and dilation angle $(\psi)$ that are having different values from cohesion $(C)$ and friction angle $(\phi)$ but having the same compressive $(\zeta)$ and tensile strength $(\xi)$. The potential function is expressed as

$$
Q(\sigma, \mathbf{q}, \mathbf{a}):=-\left[C_{q}-\tilde{\sigma}_{n n} \tan (\psi)\right]^{2} f_{c}(\sigma, \mathbf{q}, \mathbf{a}) f_{t}(\sigma, \mathbf{q}, \mathbf{a})+\tilde{\sigma}_{t t}^{2} .
$$

\section{Evolution laws}

The evolution laws describe the hardening and softening behaviour of the URM walls. The evolution laws are defined in terms of rate of plastic work per unit volume. This plastic work has different components depending upon the mode of failure it represents and is expressed in terms of internal variables which influence the yield function during plastic loading. These internal variables have been expressed as i.e. $\dot{\mathbf{W}}^{p}:=\dot{\mathbf{W}}^{p}\left(\dot{w}_{1}^{p}, \dot{w}_{2}^{p}, \dot{w}_{3}^{p}, \dot{w}_{4}^{p}\right)$ where $\dot{w}_{1}^{p}, \dot{w}_{2}^{p}$ governs the tensile strength degradation, $\dot{w}_{2}^{p}, \dot{w}_{3}^{p}$ governs the frictional strength degradation and $\dot{w}_{4}^{p}$ shows the variation in the compression strength.

$$
\begin{gathered}
\dot{w}_{1}^{p}:=\left\langle\tilde{\sigma}_{n n}\right\rangle \dot{u}_{n n}^{p} \\
\dot{w}_{2}^{p}:=\left(\tilde{\sigma}_{t t}-\tilde{\sigma}_{t t_{r 1}} \operatorname{sign}\left(\tilde{\sigma}_{t t}\right)\right) \dot{u}_{t t}^{p} \\
\dot{w}_{3}^{p}:=\left(\tilde{\sigma}_{t t_{r 1}}-\tilde{\sigma}_{t t_{r 2}}\right) \operatorname{sign}\left(\tilde{\sigma}_{t t}\right) \dot{u}_{t t}^{p} \\
\dot{w}_{4}^{p}:=\left\langle\left\langle\tilde{\sigma}_{n n}\right\rangle\right\rangle \dot{u}_{n n}^{p} .
\end{gathered}
$$

Symbols \langle\rangle and $\langle\langle\rangle\rangle$ denotes Macaulay bracket and are written as \langle\rangle$=(x+|x|) / 2$ and $\langle\langle x\rangle\rangle=(x-$ $|x|) / 2$. $\zeta_{c}$ denotes the transient point from compression cap to Mohr-Coulomb friction envelope. $\tilde{\sigma}_{t t_{r 1}}$ is the tangential strength when there is no tensile strength. $\tilde{\sigma}_{t t_{r 2}}$ is the minimum tangential strength for the new contracted yield surface. In tension-shear region $\tilde{\sigma}_{t t_{r 1}}$ and $\tilde{\sigma}_{t t_{r 2}}$ this functions is assumed to zero while in compression-shear it is expressed as

$$
\begin{aligned}
& \tilde{\sigma}_{t t_{r 1}}^{2}=-2 C_{r} \tan (\phi) f_{c} f_{t}, \\
& \tilde{\sigma}_{t t_{r 2}}^{2}=-2 C_{r} \tan (\psi) f_{c} f_{t} .
\end{aligned}
$$

These internal variables are used to calculate hardening variables $(\mathbf{q})$ and the relation is adopted from [9]. Relationship between hardening parameter $\mathbf{q}=\mathbf{q}\left(C, C_{Q}, \phi, \psi, \xi, \zeta\right)$ and internal variables is defined as:

- Cohesion

$$
C:=C_{r}+\left(C_{o}-C_{r}\right) e^{-\beta_{C}\left(\frac{w_{1}^{p}}{G_{f}^{+}}+\frac{w_{2}^{p}}{G_{f}^{H}}\right)}
$$

- Apparent Cohesion

$$
C_{Q}:=C_{Q_{r}}+\left(C_{Q_{o}}-C_{Q_{r}}\right) e^{-\beta_{C_{Q}}\left(\frac{w_{1}^{p}}{G_{f}^{I}}+\frac{w_{2}^{p}}{G_{f}^{I I}}\right)},
$$

- Friction angle

$$
\phi:=\phi_{r}+\left(\phi_{o}-\phi_{r}\right) \beta_{\phi} e^{-\beta_{\phi} w_{3}^{p}}
$$

- Dilation angle

$$
\psi:=\psi_{r}+\left(\psi_{o}-\psi_{r}\right) \beta_{\psi} e^{-\beta_{\psi} w_{3}^{p}},
$$

- Tensile strength

$$
\xi:=\xi_{o} e^{-\beta_{\tilde{\xi}}\left(\frac{w_{1}^{p}}{G_{f}^{p}}+\frac{w_{2}^{p}}{G_{f}^{I}}\right)},
$$


- Compressive strength

$$
\zeta= \begin{cases}\zeta_{o}+\left(\zeta_{p}-\zeta_{0}\right) \sqrt{\left[\frac{2 w_{4}^{p}}{w_{p}}-\left(\frac{w_{4}^{p}}{w_{p}}\right)^{2}\right]}, & \text { if } w_{4}^{p} \leq w_{p} \\ \zeta_{0}+\left(\zeta_{m}-\zeta_{p}\right)\left(\frac{w_{4}^{p}-w_{p}}{w_{m}-w_{p}}\right)^{2}, & \text { if } w_{p} \leq w_{4}^{p} \leq w_{m} \\ \zeta_{r}+\left(\zeta_{m}-\zeta_{r}\right) e^{-\beta_{\zeta}\left(\frac{w_{4}^{p}-w_{p}}{\zeta_{m}-\zeta_{r}}\right)}, & \text { if } w_{4}^{p}>w_{m}\end{cases}
$$

here $G_{f}^{I}$ and $G_{f}^{I I}$ represents the mode I and mode II fracture energy and softening of the internal variable are controlled by these parameters. Subscript $o$ refers to initial value and $r$ refers to residual value. The intermediate values are represented by subscript $p$ and $m$. The equation for hardening is written as $\dot{\mathbf{W}}^{\mathbf{p}}=\mathbf{H} \dot{\boldsymbol{e}}^{p}$.

\section{Mixed hardening law}

URM walls subjected to cyclic loading shows both kinematic and isotropic hardening behaviour during the loading, unloading and reloading cycles. The failure surface or potential function includes these hardening parameters to account for the shifts from its center and enlargements in size (see Fig. 1). Back stress vector (a) is introduced in compression cap-off $\left(f_{c}\right)$ and tensile cut-off $\left(f_{t}\right)$ functions to represent unloading/reloading behaviour. The evolution of the back stress ( $\dot{\mathbf{a}})$ is defined by Ziegler's rule to understand the behaviour of masonry under cyclic load.

$$
\dot{\mathbf{a}}=\frac{2}{3} \dot{\lambda} \boldsymbol{H} \mathbf{u}_{a}
$$

where $\dot{\lambda}$ is the rate of plastic multiplier, $\boldsymbol{H}$ is the kinematic hardening modulus, $\mathbf{u}_{a}$ is the unit vector of a. During an unloading process, whenever the stress point reaches the monotonic single yield surface then the loading surface is being controlled by the monotonic single yield surface. Whenever a reversal of stress takes place during unloading, a new unloading surface will be renewed every time. It is not activated unless the unloading surface moves and touches the monotonic yield surface or a subsequent new reversal of stress occurs. In above two cases, reversal of stress can take place before reaching the monotonic envelope, which can lead to reloading in both tension (this is similar to unloading to tension curve) and compression (this is similar to unloading to compression curve). The hardening laws presented require material parameters, which we get from experimental results of uniaxial cyclic tension and compression loading. These material parameters define the ratio between the plastic strain

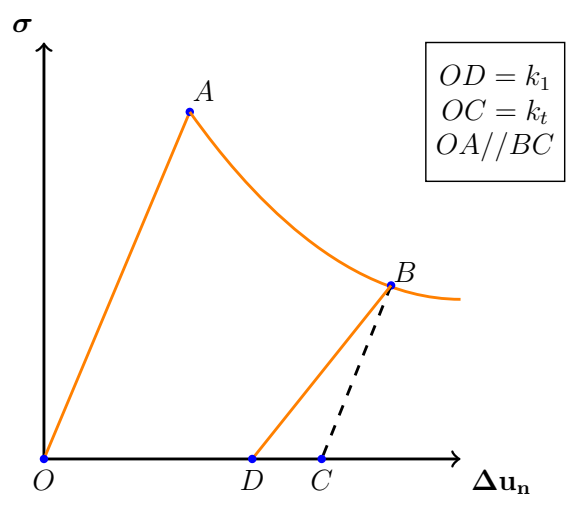

(a)

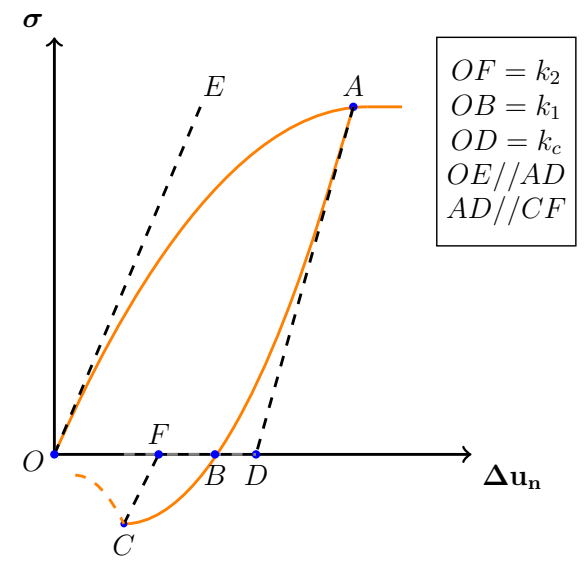

(b)

Fig. 2. Unloading points in the uniaxial stress-strain curve for (a) tensile loading (b) compression loading 
expected at some special points of the uniaxial stress-strain curve and the monotonic plastic strain. $k_{1 t}$ and $k_{1 c}$ are the points representing the plastic strain corresponding to zero stress while unloading from monotonic tensile (see Fig. 2) and compressive envelope (see Fig. 2) respectively. $k_{2 c}$ is the point which represent the plastic strain correspond to monotonic tensile envelope while unloading from the monotonic compressive envelope (see Fig. 2) and $\Delta k_{c}$ is the plastic strain increment originated by a reloading or unloading movement i.e stiffness degradation between cycles.

\section{Derivation of elasto-plastic tangent modulus}

The additive decomposition of total strain is written as

$$
\boldsymbol{\epsilon}=\boldsymbol{\epsilon}^{e}+\boldsymbol{\epsilon}^{p}
$$

where $\epsilon^{e}$ and $\epsilon^{p}$ are the elastic strain and plastic strain respectively. The direction of plastic flow is defined by flow rule.

$$
\dot{\boldsymbol{\epsilon}}^{p}=\dot{\lambda} m
$$

Kuhn Tucker conditions ( $F \leq 0, \dot{\lambda} \geq 0, F \dot{\lambda}=0$ ) provides loading and unloading conditions for plasticity based model. Plastic multiplier is determined from consistency conditions $(\dot{F} \dot{\lambda}=0)$ together with Kuhn-Tucker loading-unloading conditions. From consistency conditions, we get $\dot{F}=0$ and by solving explicitly we get plastic multiplier as follows

$$
\dot{F}=\frac{\partial F}{\partial \sigma} \dot{\sigma}+\frac{\partial F}{\partial \mathbf{q}} \dot{\mathbf{q}}+\frac{\partial F}{\partial \mathbf{a}} \dot{\mathbf{a}}=0
$$

Plastic multiplier is defined as follows

$$
\dot{\lambda}=\frac{\mathbf{n K \epsilon}}{\mathbf{n}^{T} \mathbf{k m}+\mathbf{p}^{T} \boldsymbol{\omega}_{u}+\frac{2}{3} \mathbf{s} H \mathbf{u}_{a}},
$$

where some of the terms in Eq. (18) are expressed as, $\frac{\partial F}{\partial \sigma}=\mathbf{n} ; \frac{\partial Q}{\partial \mathbf{a}}=\mathbf{s} ; \frac{\partial Q}{\partial \sigma}=\mathbf{m} ; \frac{\partial Q}{\partial \mathbf{q}}=\mathbf{p} ; \boldsymbol{\omega}_{u}=$ $\left(\frac{\partial q_{u}}{\partial \mathbf{W}^{p}}\right)\left(\frac{\partial \mathbf{W}^{p}}{\partial \epsilon^{p}}\right)\left(\frac{\partial \epsilon^{p}}{\partial \lambda}\right)$. The elasto-plastic tangent modulus is obtained by substituting the plastic multiplier in the stress-strain relation

$$
\dot{\sigma}=\mathbf{K}\left(\dot{\boldsymbol{\epsilon}}-\dot{\boldsymbol{\epsilon}}^{p}\right)=\mathbf{K}(\dot{\boldsymbol{\epsilon}}-\dot{\lambda} \mathbf{m})=\boldsymbol{K}^{e p} \dot{\boldsymbol{\epsilon}} .
$$

The elasto-plastic tangent moduli is given by

$$
\boldsymbol{K}^{e p}=\left\{\begin{array}{lll}
\mathbf{K} & \text { if } & \lambda<0 \\
\mathbf{K}-\frac{\mathbf{K m} \otimes \mathbf{n k}}{\mathbf{n}^{T} \mathbf{k} \mathbf{m}+\mathbf{p}^{T} \boldsymbol{\omega}+\frac{2}{3} \mathbf{s} \mathbf{H} \mathbf{u}_{a}} & \text { if } & \lambda>0
\end{array}\right.
$$

\section{NUMERICAL IMPLEMENTATION}

The proposed constitutive model is implemented in a finite element framework, which leads to a non-linear set of algebraic differential equations. These non-linear equations are solved by using Newton-Raphson method which leads to combination of local and global solvers to complete the numerical solution. Local solvers gives new internal state variable corresponding to relative displacement (constitutive level), subsequently global solver provides the solution for the unbalanced force to accommodate stress distribution within the finite load increments. In this section, numerical strategy is presented at both local (constitutive level) and global level (structural level).

\section{Elastic predictor-plastic corrector}

Implicit Euler backward strategy is applied to integrate differential constitutive equations . The Euler backward method is a single step method which has local truncation error of order $O\left(h^{2}\right)$ and it is unconditionally stable. These incremental equations are split into elastic predictor-plastic corrector step. Plastic corrector is applied if the current stress state violates the yield condition. Non-linear set of equations are solved by using Newton-Raphson method. The present problem is discretized in time interval $[0, T]$. Prescribed increment in the strain for the preceding time step $t_{n+1}=t_{n}+\Delta t$ is given as 
$\epsilon_{n+1}=\epsilon_{n}+\Delta \epsilon$, where $\Delta \epsilon=\Delta \epsilon_{e}+\Delta \epsilon_{p}$. In current time step $t=t_{n}$, obtained stress state must satisfy the equilibrium equations. In elastic predictor-plastic corrector step, stress and internal variables are written in incremental form.

$$
\begin{aligned}
& \sigma_{n+1}=\sigma_{n}+\mathbf{K} \Delta \boldsymbol{\epsilon}^{e}=\sigma_{n}+\mathbf{K}\left(\Delta \boldsymbol{\epsilon}-\Delta \boldsymbol{\epsilon}^{p}\right), \\
& \sigma_{n+1}=\boldsymbol{\sigma}_{n+1}^{\text {trial }}-\Delta \lambda_{n+1} \mathbf{K} \boldsymbol{m}_{n+\mathbf{1}}, \\
& \mathbf{q}_{n+1}=\mathbf{q}_{n}+\Delta \lambda_{n+1} \boldsymbol{\omega}_{n+1}, \\
& \mathbf{a}_{n+1}=\mathbf{a}_{n}+\frac{2}{3} \Delta \lambda_{n+1} \boldsymbol{H} \mathbf{u}_{a, n+1}, \\
& \kappa_{u, n+1}=\kappa_{u, n}+\Delta \lambda_{n+1}, \\
& F\left(\sigma_{n+1}, \mathbf{q}_{n+1}, \mathbf{a}_{n+1}, \kappa_{u, n+1}\right)=0 .
\end{aligned}
$$

Elastic trial step $\left(\sigma_{n+1}=\sigma_{n+1}^{\text {trial }}-\Delta \lambda_{n+1} \mathbf{K} m_{n+\mathbf{1}}\right)$ is considered over a finite step by freezing plastic step. In an elastic predictor step when the trial stress point goes outside the failure surface the plastic corrector step projects the stress at a point after the yield surface is contracted or expanded due to change in internal variable during plastic step. Therefore it is important to find a contact point at which the yield function becomes zero for the current elastic predictor step. The plastic corrector step changes the internal variables and stress. Mathematical expression for finding the contact point is expressed as

$$
F\left(\sigma_{n}+\gamma \Delta \sigma, \mathbf{q}_{n}, \mathbf{a}_{n}, \kappa_{u, n}\right)=0,
$$

where $\sigma_{n}, \mathbf{q}_{n}$, are obtained from the previously converged plastic or elastic step, and $\gamma$ is an unknown integer which varies from 0 to 1 , it converges the stress to the contact point.

\section{Local iteration strategy}

To calculate actual stress state at every load step, we need to solve the system of non-linear equations using Newton-Raphson method. The asymptotic quadratic convergence at the global level for structural equilibrium is ensured with initial root sufficiently close. The strategy is highly influenced by the choice of the independent variables and sequence of the numerical operations. It can be written as

$$
r\left(\sigma_{n+1}, \mathbf{q}_{n+1}, \mathbf{a}_{n+1}, \kappa_{n+1}, \Delta \lambda_{n+1}\right)=\left\{\begin{array}{l}
\sigma_{n+1}-\sigma_{n+1}^{t r a i l}+\Delta \lambda_{n+1} \mathbf{K m}_{n+1} \\
\mathbf{q}_{n+1}-\mathbf{q}_{n}-\Delta \lambda_{n+1} \boldsymbol{\omega}_{n+1} \\
\mathbf{a}_{n+1}-\mathbf{a}_{n}-\frac{2}{3} \Delta \lambda_{n+1} \mathbf{H} \mathbf{u}_{a, n+1} \\
\kappa_{u, n+1}-\kappa_{u, n}-\Delta \lambda_{n+1} \\
F\left(\sigma_{n+1}, \mathbf{q}_{n+1}, \mathbf{a}_{n+1}\right)=0
\end{array}\right.
$$

The nonlinear system of equations are solved by linearizing the residual as follows

$$
r(\sigma+\delta \sigma, \mathbf{q}+\delta \mathbf{q}, \mathbf{a}+\delta \mathbf{a}, \kappa+\delta \kappa, \Delta \lambda+\delta \lambda)=r(\sigma, \mathbf{q}, \mathbf{a}, \kappa, \Delta \lambda)+\frac{r(\sigma, \mathbf{q}, \mathbf{a}, \kappa, \Delta \lambda)}{\partial(\sigma, \mathbf{q}, \mathbf{a}, \kappa, \Delta \lambda)}\left\{\begin{array}{l}
\delta \sigma \\
\delta \mathbf{q} \\
\delta \mathbf{a} \\
\delta \kappa \\
\delta \lambda
\end{array}\right\}+O\left(\delta^{2}\right)
$$

In the above equation truncation after first order term is zero $O\left(\delta^{2}\right)=0$ and $\frac{r(\sigma, \mathbf{q}, \mathbf{a}, \kappa, \Delta \lambda)}{\partial(\sigma, \mathbf{q}, \mathbf{a}, \kappa, \Delta \lambda)}$ is gradient of residual term with respect to the dependent variable like $\sigma, \mathbf{q}, \mathbf{a}, \kappa, \lambda$ generally known as Jacobian. Jacobian matrix for the preceding time step $(n+1)$ is written as follows

$$
\mathbf{J}\left(\sigma_{n+1}, \mathbf{q}_{n+1}, \mathbf{a}_{n+1}, \kappa_{u, n+1}, \Delta \lambda_{n+1}\right)=\frac{r(\sigma, \mathbf{q}, \mathbf{a}, \kappa, \Delta \lambda)}{\partial(\sigma, \mathbf{q}, \mathbf{a}, \kappa, \Delta \lambda)},
$$




$$
\mathbf{J}\left(\sigma_{n+1}, \mathbf{q}_{n+1}, \mathbf{a}_{n+1}, \kappa_{u, n+1}, \Delta \lambda_{n+1}\right)=\left[\begin{array}{ccccc}
\mathbf{I}-0+\Delta \lambda \mathbf{K} \frac{\partial \mathbf{m}}{\partial \sigma} & \Delta \lambda \mathbf{K} \frac{\partial \mathbf{m}}{\partial \mathbf{q}} & \Delta \lambda \mathbf{K} \frac{\partial \mathbf{m}}{\partial \mathbf{a}} & \Delta \lambda \mathbf{K} \frac{\partial \mathbf{m}}{\partial \kappa} & \mathbf{K m} \\
-\Delta \lambda \frac{\partial \omega}{\partial \sigma} & -\Delta \lambda \frac{\partial \boldsymbol{\omega}}{\partial \mathbf{q}} & -\Delta \lambda \frac{\partial \boldsymbol{\omega}}{\partial \mathbf{a}} & -\Delta \lambda \frac{\partial \boldsymbol{\omega}}{\partial \kappa} & -\omega \\
0 & 0 & 1 & 0 & -\frac{2}{3} \Delta \lambda_{n+1} \mathbf{H u}_{a, n+1} \\
0 & 0 & 0 & 1 & 1 \\
\frac{\partial F}{\partial \sigma} & \frac{\partial F}{\partial \mathbf{q}} & \frac{\partial F}{\partial \mathbf{a}} & \frac{\partial F}{\partial \kappa} & 0
\end{array}\right]
$$

In order to get the actual solution for the current time step during plastic loading residual must be equal to zero and it is achieved by performing multiple iterations. This iteration will continue till the residual reaches the tolerance value.

$$
\begin{gathered}
0=r\left(\sigma_{n+1}^{k}, \mathbf{q}_{n+1}^{k}, \mathbf{a}_{n+1}^{k}, \kappa_{u, n+1}^{k}, \Delta \lambda_{n+1}^{k}\right)+\mathbf{J}\left(\sigma_{n+1}^{k}, \mathbf{q}_{n+1}^{k}, \mathbf{a}_{n+1}^{k}, \kappa_{u, n+1}^{k} \Delta \lambda_{n+1}^{k}\right)\left\{\begin{array}{c}
\delta \sigma_{n+1}^{k+1} \\
\delta \mathbf{q}_{n+1}^{k+1} \\
\delta \mathbf{a}_{n+1}^{k+1} \\
\delta \kappa_{u, n+1}^{k+1} \\
\delta \lambda_{n+1}^{k+1}
\end{array}\right\}, \\
\left\{\begin{array}{c}
\delta \sigma_{n+1}^{k+1} \\
\delta \mathbf{q}_{n+1}^{k+1} \\
\delta \mathbf{a}_{n+1}^{k+1} \\
\delta \kappa_{u, n+1}^{k+1} \\
\delta \lambda_{n+1}^{k+1}
\end{array}\right\}=-\mathbf{J}^{-1}\left(\sigma_{n+1}^{k}, \mathbf{q}_{n+1}^{k}, \mathbf{a}_{n+1}^{k}, \kappa_{u, n+1}^{k}, \Delta \lambda_{n+1}^{k}\right) r\left(\sigma_{n+1}^{k}, \mathbf{q}_{n+1}^{k}, \mathbf{a}_{n+1}^{k}, \kappa_{u, n+1}^{k}, \Delta \lambda_{n+1}^{k}\right), \\
\left\{\begin{array}{c}
\sigma_{n+1}^{k+1} \\
\mathbf{q}_{n+1}^{k+1} \\
\mathbf{a}_{n+1}^{k+1} \\
\kappa_{u, 1}^{k+1} \\
\lambda_{n+1}^{k+1}
\end{array}\right\}=\left\{\begin{array}{c}
\sigma_{n+1}^{k} \\
\mathbf{q}_{n+1}^{k} \\
\mathbf{a}_{n+1}^{k} \\
\kappa_{u, n+1}^{k} \\
\Delta \lambda_{n+1}^{k}
\end{array}\right\}+\left\{\begin{array}{c}
\delta \sigma_{n+1}^{k+1} \\
\delta \mathbf{q}_{n+1}^{k+1} \\
\delta \mathbf{a}_{n+1}^{k+1} \\
\delta \kappa_{u, n+1}^{k+1} \\
\delta \lambda_{n+1}^{k+1}
\end{array}\right\} .
\end{gathered}
$$

In the beginning of an iteration, initial solution has to be sufficiently close to achieve the convergence of local iteration as it depends up on the initial root. Thus initial solution is chosen to be the elastic solution at the contact point.

$$
\sigma_{n+1}^{0}=\sigma_{n+1}^{\text {trail }}+(1-\gamma) \Delta \sigma ; \quad \mathbf{q}_{n+1}^{0}=\mathbf{q}_{n} ; \quad \mathbf{a}_{n+1}^{0}=\mathbf{a}_{n} ; \quad \kappa_{u, n+1}^{0}=\kappa_{u, n} ; \quad \Delta \lambda_{n+1}^{0}=0 .
$$

During first iteration of every new load step $\Delta \lambda_{n+1}^{0}$ is equal to zero because the plastic multiplier captures the plastic process incrementally. The initial hardening parameter values $\mathbf{q}_{n+1}^{0}$ are taken from the previously converged hardening parameter values.

\section{Global iteration strategy}

The consistent tangent operator $\frac{\Delta \sigma}{\Delta \epsilon}$ is obtained from the converged solution form of local iteration strategy for the current time step. To compute the tangent operator using Jacobian, we need to differentiate the residual with respect to strain by applying chain rule as shown below

$$
\begin{gathered}
\frac{\partial}{\partial \epsilon}\left[\begin{array}{c}
\sigma_{n+1}-\boldsymbol{\sigma}_{n+1}^{\text {trail }}+\Delta \lambda_{n+1} \mathbf{K m}_{n+1} \\
\mathbf{q}_{n+1}-\mathbf{q}_{n}-\Delta \lambda_{n+1} \boldsymbol{\omega}_{n+1} \\
\mathbf{a}_{n+1}-\mathbf{a}_{n}-\frac{2}{3} \Delta \lambda_{n+1} \mathbf{H} \mathbf{u}_{a, n+1} \\
\kappa_{u, n+1}-\kappa_{u, n}-\Delta \lambda_{n+1} \\
F\left(\sigma_{n+1}, \mathbf{q}_{n+1}, \mathbf{a}_{n+1}\right)=0
\end{array}\right]=0, \\
{\left[\begin{array}{lllll}
\frac{\partial}{\partial \sigma} & \frac{\partial}{\partial \mathbf{q}} & \frac{\partial}{\partial \mathbf{a}} & \frac{\partial}{\partial \kappa_{u}} & \frac{\partial}{\partial \lambda}
\end{array}\right]_{n+1}\left[\begin{array}{c}
\sigma+\Delta \lambda \mathbf{K m} \\
\mathbf{q}-\Delta \lambda \boldsymbol{\omega} \\
\mathbf{a}-\frac{2}{3} \Delta \lambda_{n+1} \mathbf{H u} \mathbf{u}_{a, n+1} \\
\kappa_{u}-\Delta \lambda \\
\mathbf{F}(\sigma, \mathbf{q}, \mathbf{a})
\end{array}\right]_{n+1} \quad\left[\begin{array}{lllll}
\frac{\partial \sigma}{\partial \epsilon} & \frac{\partial \mathbf{q}}{\partial \epsilon} & \frac{\partial \mathbf{a}}{\partial \epsilon} & \frac{\partial \kappa_{u}}{\partial \epsilon} & \frac{\partial \lambda}{\partial \epsilon}
\end{array}\right]_{n+1}^{T}=\frac{\partial}{\partial \epsilon}\left[\begin{array}{c}
\sigma+\mathbf{K} \Delta \epsilon \\
\mathbf{q} \\
\mathbf{a} \\
\kappa_{u} \\
0
\end{array}\right]_{n}}
\end{gathered}
$$




$$
\begin{aligned}
& \mathbf{J}\left(\sigma_{n+1}^{k}, \mathbf{q}_{n+1}^{k}, \mathbf{a}_{n+1}^{k}, \kappa_{u, n+1}^{k}, \Delta \lambda_{n+1}^{k}\right)\left[\begin{array}{lllll}
\frac{\partial \sigma}{\partial \boldsymbol{\epsilon}} & \frac{\partial \mathbf{q}}{\partial \boldsymbol{\epsilon}} & \frac{\partial \mathbf{a}}{\partial \boldsymbol{\epsilon}} & \frac{\partial \kappa_{u}}{\partial \boldsymbol{\epsilon}} & \frac{\partial \lambda}{\partial \boldsymbol{\epsilon}}
\end{array}\right]_{n+1}^{T}=\left[\begin{array}{c}
\mathbf{K} \\
0 \\
0 \\
0 \\
0
\end{array}\right], \\
& {\left[\begin{array}{l}
{\left[\frac{\partial \sigma}{\partial \boldsymbol{\epsilon}}\right]_{2 \times 2}} \\
{\left[\frac{\partial \mathbf{q} u}{\partial \boldsymbol{\epsilon}}\right]_{6 \times 2}} \\
{\left[\frac{\partial \mathbf{a}}{\partial \boldsymbol{\epsilon}}\right]_{1 \times 2}} \\
{\left[\frac{\partial \kappa}{\partial \boldsymbol{\epsilon}}\right]_{1 \times 2}} \\
{\left[\frac{\partial \lambda}{\partial \boldsymbol{\epsilon}}\right]_{1 \times 2}}
\end{array}\right]_{11 \times 2}} \\
& =\mathbf{J}^{-1}\left(\boldsymbol{\sigma}_{n+1}, \mathbf{q}_{n+1}, \mathbf{a}_{n+1}, \kappa_{u, n+1}, \Delta \lambda_{n+1}\right) 11 \times 2\left[\begin{array}{c}
\mathbf{K} \\
0 \\
0 \\
0 \\
0
\end{array}\right]_{11 \times 2} .
\end{aligned}
$$

Consistent tangent operator is defined from the above equation as follows:

$$
\left[\frac{\partial \sigma}{\partial \boldsymbol{\epsilon}}\right]_{2 \times 2}=O_{2 \times 2}\left\{\mathbf{J}^{-1}\left(\sigma_{n+1}, \mathbf{q}_{n+1}, \mathbf{a}_{n+1}, \Delta \lambda_{n+1}\right)_{11 \times 11}\left[\begin{array}{c}
\mathbf{K} \\
0 \\
0 \\
0
\end{array}\right]_{11 \times 2}\right\} .
$$

\section{Algorithm}

The set of non-linear equations are solved by using Newton-Raphson method by a combined local and global approach. The numerical model has been implemented in ABAQUS [28] by writing a UMAT subroutine, which allows user to define the material constitutive model. During analysis UMAT will be called at all integration points of the element and it will update internal state variables, elasto-plastic tangent operator and stress increment at the end of the increment. The algorithm used to implement the proposed constitutive model in UMAT is presented as follows:

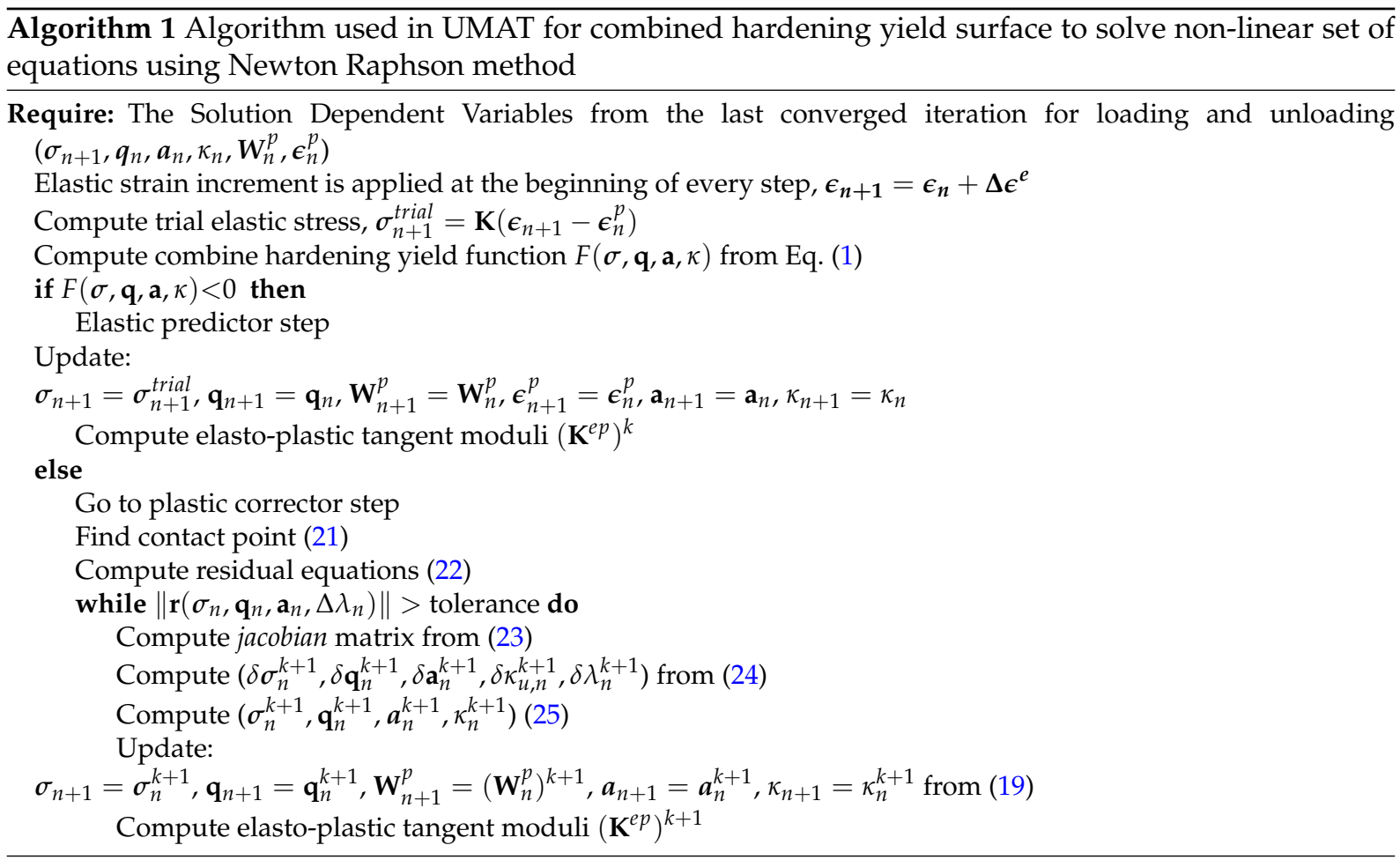




\section{NUMERICAL EXAMPLES}

\subsection{Two-brick-mortar interface subjected to cyclic loading}

Two-brick lay up with a mortar interface is considered for the analysis. The modelling of the two-brick model with interface element is done in ABAQUS and the proposed constitutive model is implemented by using user defined material (UMAT) for simulating the behaviour under monotonic and cyclic loading in both tension and compression. The expanded units are modelled using bilinear quadrilateral plane stress element (CPS4R) elements and brick-mortar interface is modelled as zero thickness cohesive element (COH2D4). Experimental results on masonry wall $[29,30]$ show that crack either passes through mortar or through the middle of the brick, therefore bed and head mortar joint interface are modelled as zero-thickness interface elements and each brick is divided into two parts with an interface element in the middle.

Gopalaratnam and Shah [31] have conducted experiments on concrete specimens under cyclic tension. Material parameters adopted for the present model are obtained indirectly from calibration process as shown in Tabs. 1, 2 and 3. The obtained numerical results from the proposed model for a two-brick model under cyclic tension are compared with the experimental results as shown in Fig. 3. The numerical results obtained from the present model is found to match well with experimental results in terms of strength and stiffness degradation.

Table 1. Elastic properties of the bricks and joints (tensile loading)

\begin{tabular}{cccc}
\hline$E\left(\mathrm{~N} / \mathrm{mm}^{2}\right)$ & $\gamma$ & $k_{n n}\left(\mathrm{~N} / \mathrm{mm}^{3}\right)$ & $k_{t t}\left(\mathrm{~N} / \mathrm{mm}^{3}\right)$ \\
\hline 16,700 & 0.15 & 82 & 36 \\
\hline
\end{tabular}

Table 2. Monotonic inelastic properties of the joints (tension loading)

\begin{tabular}{ccccccc}
\hline Tension & \multicolumn{5}{c}{ Shear } \\
\hline$\xi_{o}\left(\mathrm{~N} / \mathrm{mm}^{2}\right)$ & $G_{f}^{1}\left(\mathrm{Nmm} / \mathrm{mm}^{2}\right)$ & $C_{o} \backslash C_{r}$ & $C_{q o} \backslash C_{q r}$ & $\phi_{o} \backslash \phi_{r}$ & $\psi_{o} \backslash \psi_{r}$ & $G_{f}^{2}\left(\mathrm{Nmm} / \mathrm{mm}^{2}\right)$ \\
\hline 2 & $5 \frac{\xi_{o}^{2}}{2 * K_{n n}}$ & $2.8 \backslash 0.28$ & $2.2 \backslash 0.22$ & $0.7 \backslash 0.6$ & $0.3 \backslash 0.2$ & $10^{*} G_{f}^{1}$ \\
\hline
\end{tabular}

Table 3. Monotonic inelastic properties of the joints (tensile loading)

\begin{tabular}{cccccc}
\hline Cap & & & & \\
\hline$\zeta_{o} \backslash \zeta_{p}\left(\mathrm{~N} / \mathrm{mm}^{2}\right)$ & $\zeta_{m} \backslash \zeta_{f}\left(\mathrm{~N} / \mathrm{mm}^{2}\right)$ & $\beta_{c} \backslash \beta_{c q}$ & $\beta_{\phi} \backslash \beta_{p s i}$ & $\beta_{\zeta} \backslash \beta_{\zeta 1}$ & $\alpha_{1} \backslash \alpha_{2}$ \\
\hline$-20 \backslash-30$ & $-22 \backslash-5$ & $0.12 \backslash 0.12$ & $0.02 \backslash 0.02$ & $0.12 \backslash 5$ & $4.5 \backslash 4.5$ \\
\hline
\end{tabular}

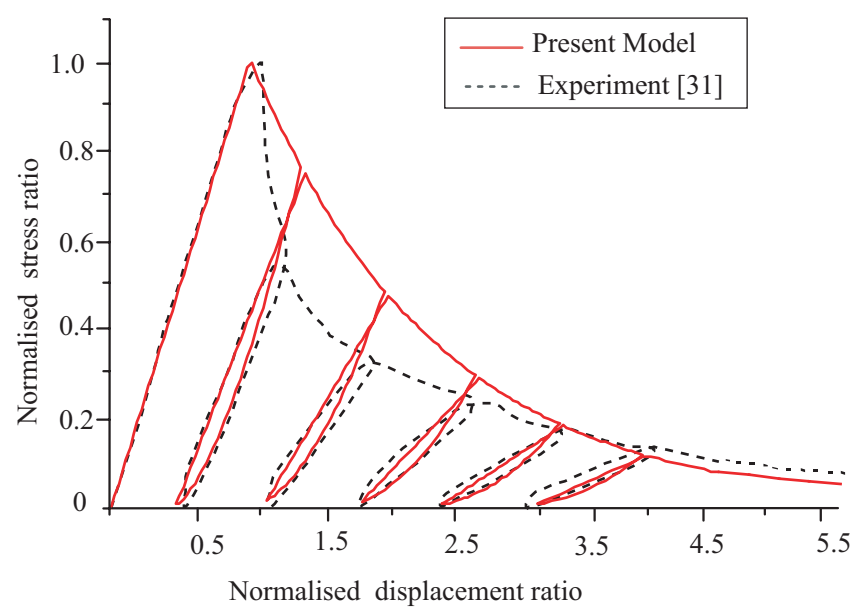

Fig. 3. Comparision of the present model with experimental results under cyclic tensile load 
Similarly for cyclic compression loading the results are compared with the experimental results. The present model is able to simulate the behaviour of two-brick model under cyclic compression loading. Material parameters adopted for this example are shown in Tabs. 4, 5 and 6. The obtained numerical results of the present model is compared with the experimental results conducted by Karsan and Jirsa [32] (see Fig. 4). The obtained numerical results is in good agreement with the experimental values in terms of stiffness and strength degradation. The present constitutive model is able to capture the stiffness and strength degradation during each unloading/reloading cycle and between the cycles for tensile and compression cyclic loading.

Table 4. Elastic properties of the bricks and joints (compression loading)

\begin{tabular}{cccc}
\hline$E\left(\mathrm{~N} / \mathrm{mm}^{2}\right)$ & $\gamma$ & $k_{n n}\left(\mathrm{~N} / \mathrm{mm}^{3}\right)$ & $k_{t t}\left(\mathrm{~N} / \mathrm{mm}^{3}\right)$ \\
\hline 16,700 & 0.15 & 110 & 50 \\
\hline
\end{tabular}

Table 5. Inelastic properties of the joints (compression loading)

\begin{tabular}{|c|c|c|c|c|c|c|}
\hline Tension & & & & Shear & & \\
\hline$\xi_{o}\left(\mathrm{~N} / \mathrm{mm}^{2}\right)$ & $\mathrm{G}_{f}^{1}\left(\mathrm{Nmm} / \mathrm{mm}^{2}\right)$ & $C_{o} \backslash C_{r}$ & $C_{q o} \backslash C_{q r}$ & $\phi_{o} \backslash \phi_{r}$ & $\psi_{o} \backslash \psi_{r}$ & $G_{f}^{2}\left(\mathrm{Nmm} / \mathrm{mm}^{2}\right)$ \\
\hline 2 & $5 \frac{\xi_{0}^{2}}{2 * K_{n n}}$ & $2.8 \backslash 0.28$ & $2.2 \backslash 0.22$ & $0.7 \backslash 0.6$ & $0.3 \backslash 0.2$ & $10^{*} G_{f}^{1}$ \\
\hline
\end{tabular}

Table 6. Inelastic properties of the joints (compression loading)

\begin{tabular}{cccccc}
\hline Cap & & & & \\
\hline$\zeta_{o} \backslash \zeta_{p}\left(\mathrm{~N} / \mathrm{mm}^{2}\right)$ & $\zeta_{m} \backslash \zeta_{f}\left(\mathrm{~N} / \mathrm{mm}^{2}\right)$ & $\beta_{c} \backslash \beta_{c q}$ & $\beta_{\phi} \backslash \beta_{p s i}$ & $\beta_{\zeta} \backslash \beta_{\zeta 1}$ & $\alpha_{1} \backslash \alpha_{2}$ \\
\hline$-20 \backslash-30$ & $-22 \backslash-5$ & $0.12 \backslash 0.12$ & $0.02 \backslash 0.02$ & $0.12 \backslash 5$ & $4.5 \backslash 4.5$ \\
\hline
\end{tabular}

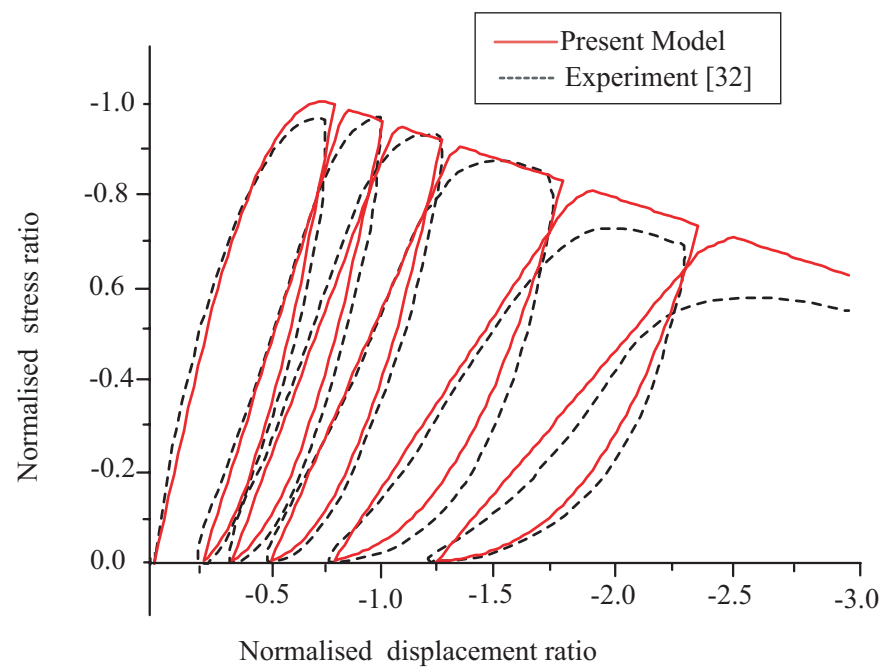

Fig. 4. Comparison of the present model with experimental results under cyclic compression load

The two brick lay up is subjected to cyclic shear loads. The obtained numerical results of the proposed model is able to describe the behavior of two brick model subjected to cyclic shear loading by assuming elastic unloading/reloading behaviour. Atkinson et al. [11] conducted direct shear test on mortar joints to capture shear behaviour of mortar subjected to cyclic shear loading. The numerical results from the present study are validated by comparing with the experimental results (see Fig. 5). 


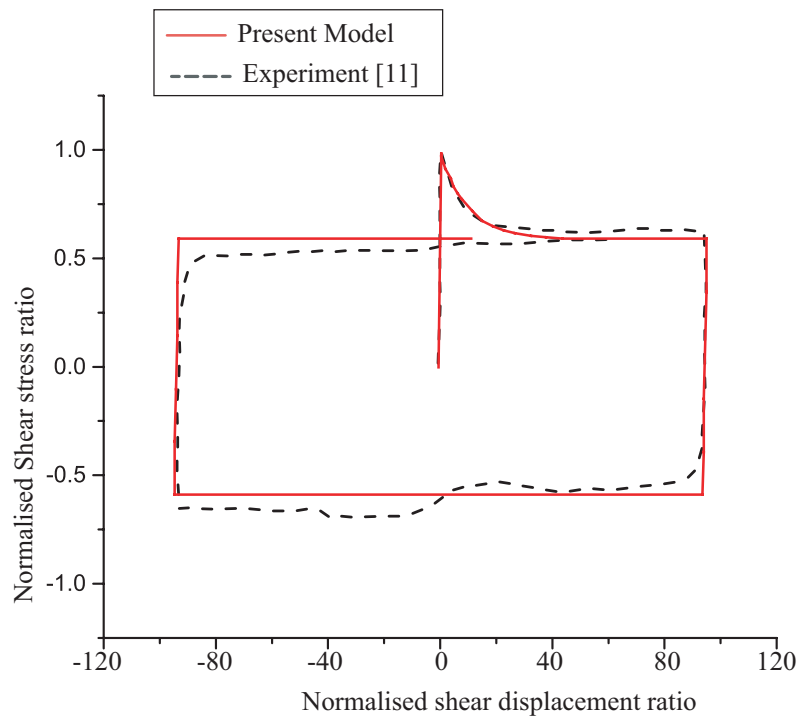

Fig. 5. Comparison of the present model with experimental results under cyclic shear load

Table 7. Cylic material properties for two-brick model

\begin{tabular}{ccccc}
\hline Numerical simulation & $\frac{\kappa_{1}}{\kappa_{t}}$ & $\frac{\kappa_{1}}{\kappa_{c}}$ & $\frac{\kappa_{2}}{\kappa_{c}}$ & $\frac{\Delta \kappa_{c}}{\kappa_{\mathcal{c}}}$ \\
\hline Gopalaratnam and Shah [31] & 0.76 & - & - & - \\
Karsan and Jirsa [32] & - & 0.56 & 0.28 & 0.13 \\
Reinhardt [33] & 0.73 & - & - & - \\
\hline
\end{tabular}

The two brick lay up is subjected to cyclic tension-compression loading. The obtained numerical results of the proposed model is able to describe the behavior of two brick model under six cycles of load reversal. Reinhardt [33] performed experiments on concrete specimens under tension-compression loading. The obtained numerical results is matching well with the experimental results in terms of strength and stiffness degradation associated with reopening and closing of cracks (see Fig. 6) between the cycles. Tab. 7 shows the cyclic material parameters adopted in the present model to numerically simulate the two-brick for various cyclic loading cases.

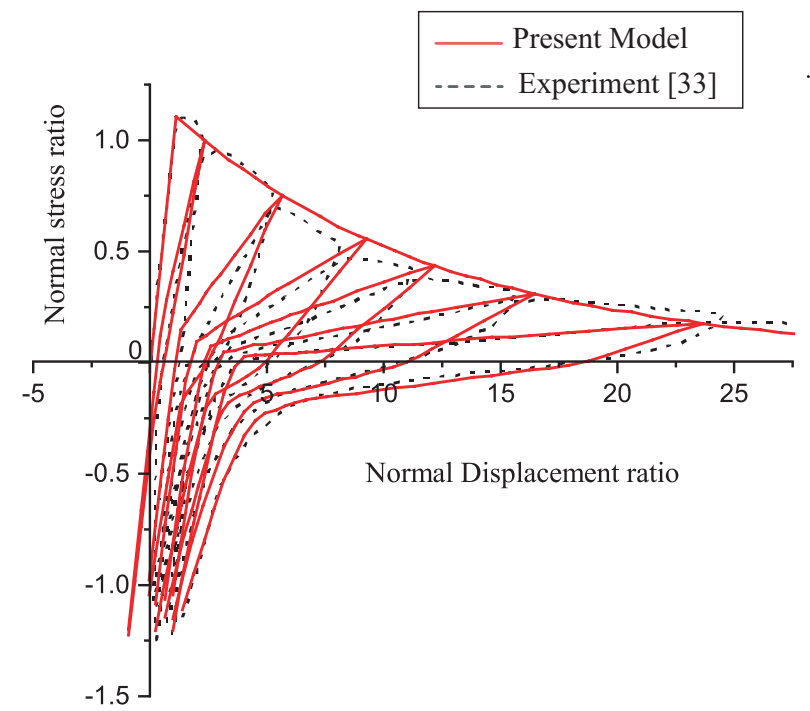

Fig. 6. Comparison of the present model with experimental results under cyclic tension-compression load 


\subsection{URM shear wall subjected to cyclic loading}

Vermeltfoort and Raijmakers [29,30] conducted experiments on masonry shear wall subjected to monotonic loading. The same experimental setup is used for the numerical modelling of the URM shear wall (see Fig. 7) in ABAQUS, where brick units are modelled using bilinear quadrilateral plane stress element (CPS4R) with four degrees of freedom at each node and brickmortar interface are modelled as zero thickness cohesive element (COH2D4). Elastic properties of URM wall for brick and joints are shown in Tab. 8. Tab. 9 shows cyclic inelastic properties of the joints for URM wall. These properties are adopted from Oliveria et al. [22]. A vertical uniform stress of $1.21 \mathrm{MPa}$ is ap-

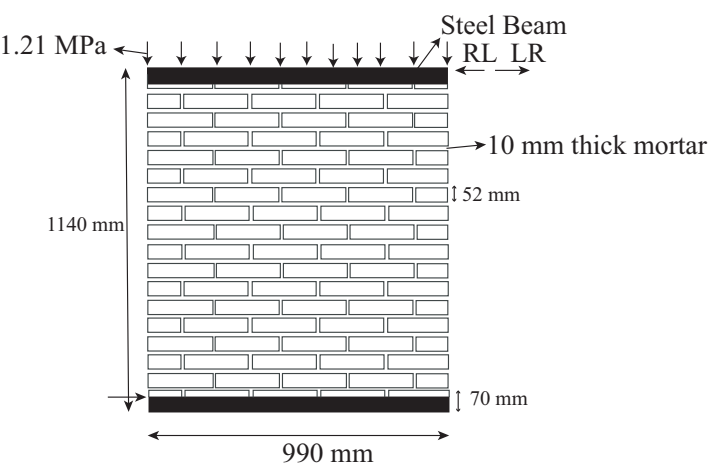

Fig. 7. Geometry of tested TUE unreinforced masonry wall plied on the wall panel along with varying in-plane cyclic horizontal displacement of $1 \mathrm{~mm}, 2 \mathrm{~mm}$, $3 \mathrm{~mm}$ and $4 \mathrm{~mm}$ applied on the wall panel consecutively and unloading is performed till it attains zero horizontal force.

Table 8. Elastic properties of the bricks and joints (TUE wall)

\begin{tabular}{cccc}
\hline$E\left(\mathrm{~N} / \mathrm{mm}^{2}\right)$ & $\gamma$ & $k_{n n}\left(\mathrm{~N} / \mathrm{mm}^{3}\right)$ & $k_{t t}\left(\mathrm{~N} / \mathrm{mm}^{3}\right)$ \\
\hline 16700 & 0.15 & 110 & 50 \\
\hline
\end{tabular}

Table 9. Cylic inelastic properties of the joints (TUE wall)

\begin{tabular}{cccc}
\hline Tension & \multicolumn{2}{c}{ Tension-compression } & Compression \\
\hline$\frac{\kappa_{1}}{\kappa_{t}}$ & $\frac{\kappa_{1}}{\kappa_{c}}$ & $\frac{\kappa_{2}}{\kappa_{c}}$ & $\frac{\Delta \kappa_{c}}{\kappa_{c}}$ \\
\hline 0.60 & 0.75 & 0.50 & 0.0 \\
\hline
\end{tabular}

In each cycle, unloading is done in a linear fashion to capture the stiffness degradation. While initially reloading, it will show high stiffness due to closing of diagonal shear cracks and then the stiffness decreases progressively for subsequent cycles. Fig. 8 show the numerical horizontal load-displacement

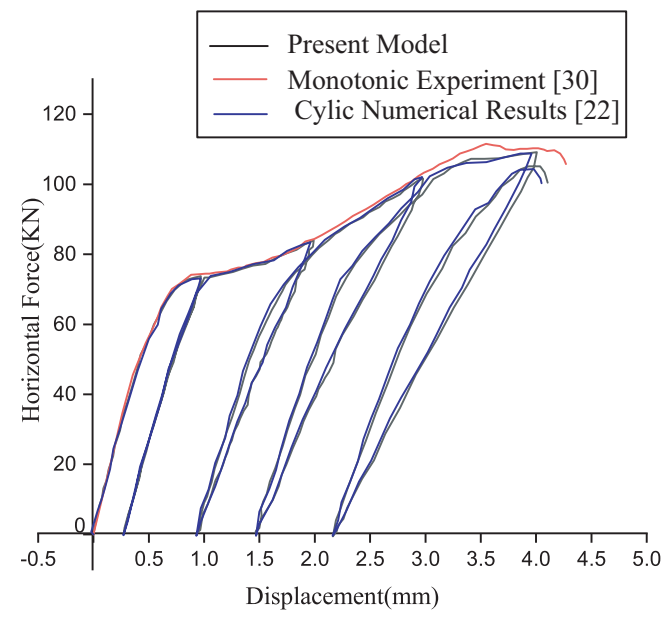

(a)

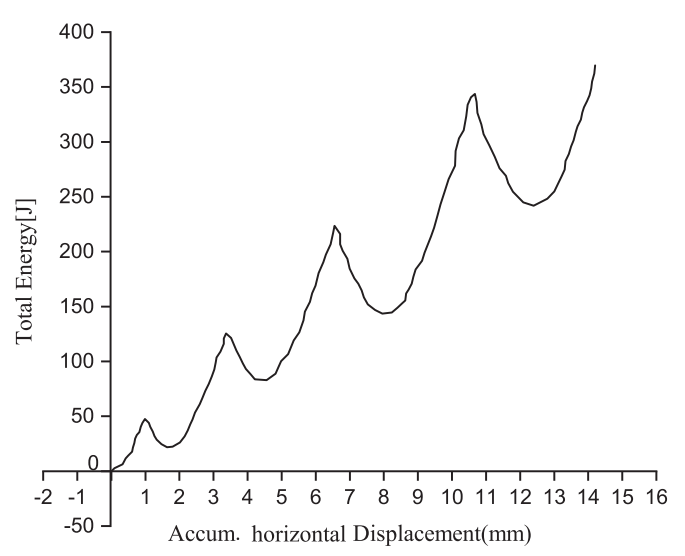

(b)

Fig. 8. URM wall (a) Comparison of the cyclic full compression horizontal load-displacement graph of the present model with monotonic experiment and cyclic numerical results of Oliveria et al. [22] (b) Total energy evolution 
graph and total energy dissipated-accumulated displacement graph of the URM wall subjected to cyclic compression loading. The principal compressive stress corresponding to $4 \mathrm{~mm}$ and unloading from $4 \mathrm{~mm}$ to zero force are shown in Fig. 9. Two compressive struts are developed (see Fig. 9) due to evolution of diagonal crack that characterizes the structural behaviour. Fig. 10 shows the numerical horizontal load-displacement graph and energy dissipation for the masonry wall subjected to cyclic tension-compression loading. The obtained numerical results (see Figs. 8 and 10) are validated with monotonic experimental results [29,30] and with Oliveria et al. [22] model available in the literature. As an indication shown in the experimental studies, the diagonal shear cracks are formed and thereupon reduces the stiffness and strength. The failure in the URM wall is shear dominating, which primarily accounts for decrease in strength. While unloading cracked head joints are closing partially and bed joints slide in the opposite direction. Softening behaviour of masonry wall is due to compression crushing failure at lower toe of the wall which leads to failure.

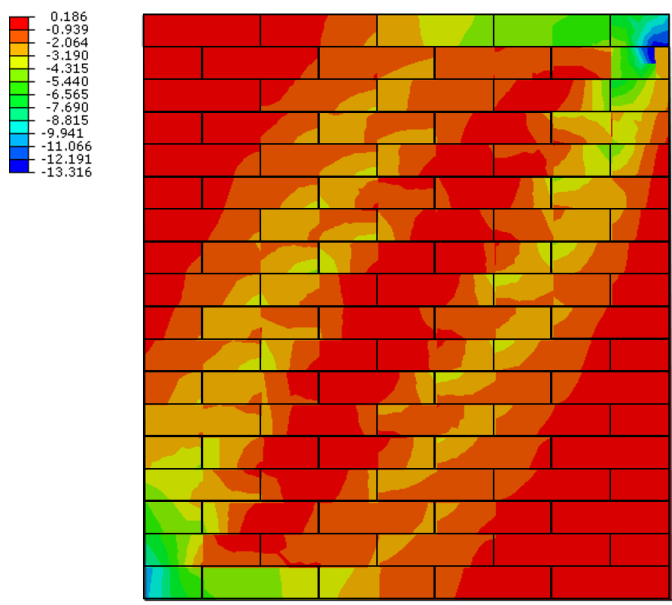

(a)

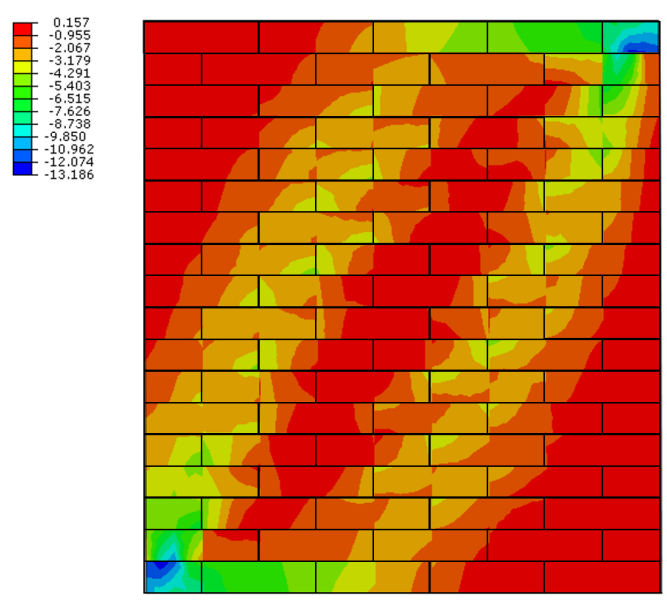

(b)

Fig. 9. Principal compressive stress [N/ $\left.\mathrm{mm}^{2}\right]$ for a horizontal displacement (a) $4 \mathrm{~mm}$ (b) unloading from $4 \mathrm{~mm}$ to zero horizontal force

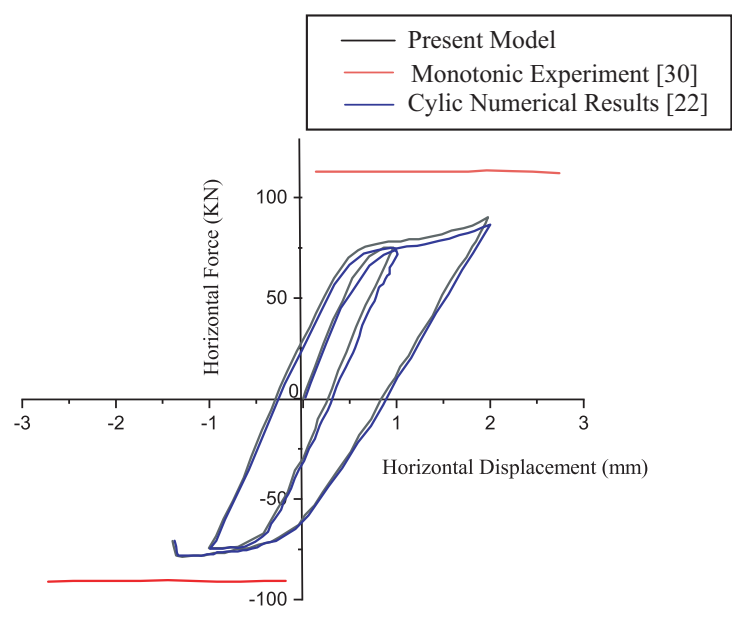

(a)

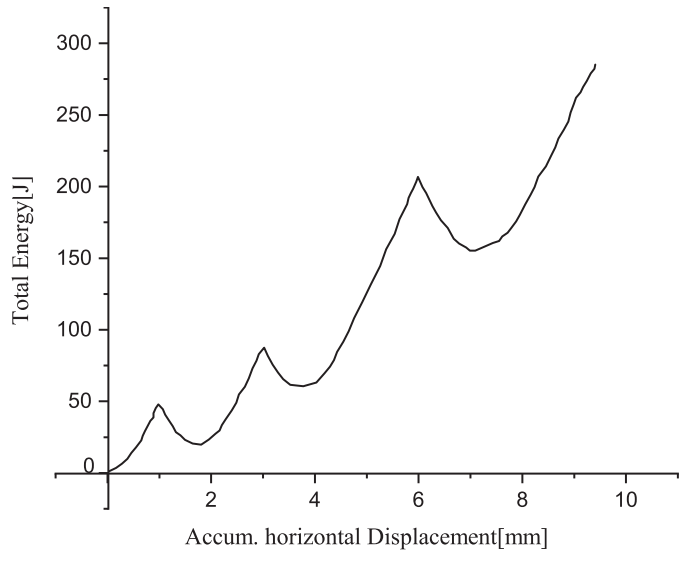

(b)

Fig. 10. URM wall (a) Comparison of the cyclic tension-compression horizontal load-displacement graph of the present model with monotonic experiment and cyclic numerical results of Oliveria et al. [22]

(b) Total energy evolution 


\section{SUMMARY AND CONCLUSIONS}

The proposed plasticity based interface model is able to describe the failure behaviour of URM under cyclic loading. This is achieved by introducing a back stress vector as a mixed hardening law variable in the single yield surface to capture unloading-reloading behaviour of masonry under cyclic loading. In this model, linear unloading is assumed for shear component whereas non-linear form is assumed for normal component of stress. The present plasticity based interface model is applied to two-brick model and URM subjected to various loading. The present modification of numerical model captures the non-linear behaviour of URM which is able to reproduce opening-closure and shear sliding of joints, energy dissipation, stiffness/strength degradation and diagonal shear failure patterns similar to experimental results reported in the literature.

\section{REFERENCES}

[1] A. M. D'Altri, F. Messali, J. Rots, G. Castellazzi, and S. de Miranda. A damaging block-based model for the analysis of the cyclic behaviour of full-scale masonry structures. Engineering Fracture Mechanics, 209, (2019), pp. 423-448. https://doi.org/10.1016/j.engfracmech.2018.11.046.

[2] M. Shadlou, E. Ahmadi, and M. M. Kashani. Micromechanical modelling of mortar joints and brick-mortar interfaces in masonry Structures: A review of recent developments. Structures, 23, (2020), pp. 831-844. https://doi.org/10.1016/j.istruc.2019.12.017.

[3] L. Macorini and B. A. Izzuddin. A non-linear interface element for 3D mesoscale analysis of brickmasonry structures. International Journal for numerical methods in Engineering, 85, (12), (2011), pp. 1584-1608. https://doi.org/10.1002/nme.3046.

[4] C. Calderini and S. Lagomarsino. Continuum model for in-plane anisotropic inelastic behavior of masonry. Journal of Structural Engineering, 134(2), (2008), pp. 209-220. https://doi.org/10.1061/(asce)07339445(2008)134:2(209).

[5] E. Minga, L. Macorini, and B. A. Izzuddin. A 3D mesoscale damage-plasticity approach for masonry structures under cyclic loading. Meccanica, 53, (7), (2018), pp. 1591-1611. https://doi.org/10.1007/s11012-017-0793-z.

[6] A. M. D'Altri, S. de Miranda, G. Castellazzi, and V. Sarhosis. A 3D detailed micro-model for the in-plane and out-of-plane numerical analysis of masonry panels. Computers \& Structures, 206, (2018), pp. 18-30. https://doi.org/10.1016/j.compstruc.2018.06.007.

[7] E. Bertolesi, G. Milani, and P. B. Lourenco. Implementation and validation of a total displacement nonlinear homogenization approach for in-plane loaded masonry. Computers and Structures, 176, (2016), pp. 13-33. https://doi.org/10.1016/j.compstruc.2016.08.001.

[8] K. F. Abdulla, L. S. Cunningham, and M. Gillie. Simulating masonry wall behaviour using a simplified micro-model approach. Engineering Structures, 151, (2017), pp. 349-365. https://doi.org/10.1016/j.engstruct.2017.08.021.

[9] N. Kumar, A. Rajagopal, and M. Pandey. Plasticity based approach for failure modelling of unreinforced masonry. Engineering Structures, 80, (2014), pp. 40-52. https://doi.org/10.1016/j.engstruct.2014.08.021.

[10] P. B. Lourenco and J. G. Rots. Multisurface interface model for analysis of masonry structures. Journal of Engineering Mechanics, 123, (7), (1997), pp. 660-668. https://doi.org/10.1061/(asce)0733-9399(1997)123:7(660).

[11] A. D. Jefferson and N. R. Mills. Fracture and shear properties of concrete construction joints from core samples. Materials and Structures, 31, (1998), pp. 595-601. https://doi.org/10.1007/bf02480609.

[12] P. B. Lourenco and L. F. Ramos. Characterization of cyclic behavior of dry masonry joints. Journal of Structural Engineering, 130, (5), (2004), pp. 779-786. https://doi.org/10.1061/(asce)0733-9445(2004)130:5(779).

[13] R. H. Atkinson, B. P. Amadei, S. Saeb, and S. Sture. Response of masonry bed joints in direct shear. Journal of Structural Engineering, 115, (1989), pp. 2276-2296. https://doi.org/10.1061/(asce)0733-9445(1989)115:9(2276).

[14] L. C. Silva, P. B. Lourenco, and G. Milani. Numerical homogenization-based seismic assessment of an Englishbond masonry prototype: Structural level application. Earthquake Engineering E Structural Dynamics, 49, (9), (2020), pp. 841-862. https://doi.org/10.1002/eqe.3267.

[15] T. Y. P. Yuen, T. Deb, H. Zhang, and Y. Liu. A fracture energy based damage-plasticity interfacial constitutive law for discrete finite element modelling of masonry structures. Computers and Structures, 220, (2019), pp. 92 113. https://doi.org/10.1016/j.compstruc.2019.05.007.

[16] D. Liberatore, D. Addessi, and M. Sangirardi. Nonlinear analysis of masonry walls based on a damage-plastic formulation. Structural Analysis of Historical Constructions, 18, (2019), pp. 1009-1017. https://doi.org/10.1007/978-3-319-99441-3_109.

[17] F. Parrinello, B. Failla, and G. Borino. Cohesive frictional interface constitutive model. International Journal of Solids and Structures, 46, (2009), pp. 2680-2692. https://doi.org/10.1016/j.ijsolstr.2009.02.016. 
[18] T. Stankowski, K. Runesson, and S. Sture. Fracture and slip of interfaces in cementitious composites. I: Characteristics. Journal of Engineering Mechanics, 119, (2), (1993), pp. 292-314. https://doi.org/10.1061/(asce)07339399(1993)119:2(292).

[19] V. G. Haach, G. Vasconcelos, and P. B. Lourenco. Parametrical study of masonry walls subjected to in-plane loading through numerical modeling. Engineering Structures, 33, (4), (2011), pp. 1377-1389. https://doi.org/10.1016/j.engstruct.2011.01.015.

[20] S. Nazir and M. Dhanasekar. Modelling the failure of thin layered mortar joints in masonry. Engineering Structures, 49, (2013), pp. 615-627. https://doi.org/10.1016/j.engstruct.2012.12.017.

[21] D. Addessi and E. Sacco. Nonlinear analysis of masonry panels using a kinematic enriched plane state formulation. International Journal of Solids and Structures, 90, (2016), pp. 194-214. https://doi.org/10.1016/j.ijsolstr.2016.03.002.

[22] D. V. Oliveira and P. B. Lourenco. Implementation and validation of a constitutive model for the cyclic behaviour of interface elements. Computers and Structures, 82, (2004), pp. 1451-1461. https://doi.org/10.1016/s0045-7949(04)00131-2.

[23] A. M. D'Altri, F. Messali, J. Rots, G. Castellazzi, and S. de Miranda. A damaging block-based model for the analysis of the cyclic behaviour of full-scale masonry structures. Engineering Fracture Mechanics, 209, (2019), pp. 423-448. https://doi.org/10.1016/j.engfracmech.2018.11.046.

[24] P. C. Miglietta, E. C. Bentz, and G. Grasselli. Finite/discrete element modelling of reversed cyclic tests on unreinforced masonry structures. Engineering Structures, 138, (2017), pp. 159-169. https://doi.org/10.1016/j.engstruct.2017.02.019.

[25] C. Gatta, D. Addessi, and F. Vestroni. Static and dynamic nonlinear response of masonry walls. International Journal of Solids and Structures, 155, (2018), pp. 291-303. https://doi.org/10.1016/j.ijsolstr.2018.07.028.

[26] N. Mojsilovic, M. Petrovic, and X. R. Anglada. Masonry elements with multi-layer bed joints: Behaviour under monotonic and static-cyclic shear. Construction and Building Materials, 100, (2016), pp. 149-162. https://doi.org/10.1016/j.conbuildmat.2015.09.065.

[27] A. J. Aref and K. M. Dolatshahi. A three-dimensional cyclic meso-scale numerical procedure for simulation of unreinforced masonry structures. Computers and Structures, 120, (2013), pp. 9-23. https://doi.org/10.1016/j.compstruc.2013.01.012.

[28] Abaqus 6.14 online documentation. SIMULIA Inc.

[29] T. M. J. Raijmakers and A. T. Vermeltfoort. Deformation controlled tests in masonry shear walls. Technical report, TNO-Bow, Delft, The Netherlands, (1992).

[30] A. T. Vermeltfoort and T. M. J. Raijmakers. Deformation controlled tests in masonry shear walls, Part 2. Technical report, Eindhoven University of Technology, Eindhoven, The Netherlands, (1993).

[31] V. S. Gopalaratnam and S. P. Shah. Softening response of plain concrete in direct tension. Journal Proceedings, American Concrete Institute, 82(3), (1985), pp. 310-323.

[32] I. D. Karsan and J. O. Jirsa. Behavior of concrete under compressive loadings. Journal of Structural Division, ASCE, 95, (1969), pp. 2543-2563.

[33] H. W. Reinhardt. Fracture mechanics of an elastic softening material like concrete. Heron, 29 (2), (1984), pp. 341. 